\title{
Generalized Derivations and Generalization of Co-commuting Maps in Prime Rings
}

\author{
Basudeb Dhara*, Nripendu Bera, Sukhendu Kar and Brahim Fahid
}

\begin{abstract}
Suppose that $R$ is a prime ring of characteristic different from 2 with Utumi quotient ring $U, C=Z(U)$ the extended centroid of $R$, and $f\left(x_{1}, \ldots, x_{n}\right)$ a noncentral multilinear polynomial over $C$. If $F, G$ and $H$ are three nonzero generalized derivations of $R$ such that

$$
F(G(f(X)) f(X))=f(X) H(f(X))
$$

for all $X=\left(x_{1}, \ldots, x_{n}\right) \in R^{n}$, then we describe the nature of the maps $F, G$ and $H$.
\end{abstract}

\section{Introduction}

Throughout this paper $R$ denotes a prime ring with center $Z(R)$, extended centroid $C$ and $U$ its Utumi quotient ring. The definition and axiomatic formulation of Utumi quotient ring $U$ can be found in 2, 4].

We have the following properties which we need

1. $R \subseteq U$;

2. $U$ is a prime ring with unity;

3. The center of $U$ is denoted by $C$ and is called the extended centroid of $R$. $C$ is a field.

By a derivation of $R$, we mean an additive mapping $d: R \rightarrow R$ such that $d(x y)=$ $d(x) y+x d(y)$ holds for all $x, y \in R$. An additive mapping $F: R \rightarrow R$ is called a generalized derivation if there exists a derivation $d$ on $R$ such that $F(x y)=F(x) y+x d(y)$ holds for all $x, y \in R$. Basic examples of generalized derivations are derivations, generalized inner derivations (i.e., maps of type $x \rightarrow a x+x b$ for some $a, b \in R$ ). In [16], Lee proved that any generalized derivation of $R$ can be uniquely extended to a generalized derivation of $U$ and its form will be $g(x)=a x+d(x)$ for some $a \in U$, where $d$ is the Received May 13, 2020; Accepted August 2, 2020.

Communicated by Kunio Yamagata.

2010 Mathematics Subject Classification. 16W25, 16N60.

Key words and phrases. prime ring, derivation, generalized derivation, extended centroid, Utumi quotient ring.

*Corresponding author. 
associated derivation. The Lie commutator of $x$ and $y$ is denoted by $[x, y]$ and also defined by $[x, y]=x y-y x$ for all $x, y \in R$; also the symbol $x \circ y$ stands for the anticommutator $x y+y x$. By $s_{4}$, we denotes the standard polynomial in four variables, which is $s_{4}\left(x_{1}, x_{2}, x_{3}, x_{4}\right)=\sum_{\sigma \in S_{4}}(-1)^{\sigma} x_{\sigma(1)} x_{\sigma(2)} x_{\sigma(3)} x_{\sigma(4)}$, where $(-1)^{\sigma}$ is +1 or -1 according to $\sigma$ being an even or odd permutation in symmetric group $S_{4}$. Let $S \subseteq R$. An additive map $F: R \rightarrow R$ is said to be commuting (centralizing) on $S$ if $[F(x), x]=0$ for all $x \in S$ (resp. $[F(x), x] \in Z(R)$ for all $x \in S$ ). Two additive maps $F, G: R \rightarrow R$ are said to be co-commuting (co-centralizing) on $S$ if $F(x) x-x G(x)=0$ for all $x \in S$ (resp. $F(x) x-x G(x) \in Z(R)$ for all $x \in S$ ).

In [6], De Filippis and De Vincenzo described the structure of additive mappings $d$ and $G$ satisfying $d(G(f(X)) f(X)-f(X) G(f(X)))=0$ for all $X=\left(x_{1}, \ldots, x_{n}\right) \in R^{n}$, where $f$ is a multinear polynomial over extended centroid $C$ and $d$ is a nonzero derivation and $G$ is a nonzero generalized derivation on prime $\operatorname{ring} R$ of $\operatorname{char}(R) \neq 2$.

In [9], the first author, Argac and Albas extended the above result by considering two generalized derivations. More precisely, they studied the situation $d(F(f(X)) f(X)-$ $f(X) G(f(X)))=0$ for all $X=\left(x_{1}, \ldots, x_{n}\right) \in R^{n}$, where $f$ is a multinear polynomial over extended centroid $C$ and $d$ is a nonzero derivation and $F, G$ are two generalized derivations on prime $\operatorname{ring} R$ of $\operatorname{char}(R) \neq 2$. In the paper authors determined all possible forms of the additive maps $d, F$ and $G$.

On the other hand, Carini and De Filippis 3 proved that if $R$ is a prime ring of characteristic different from $2, \delta$ a nonzero derivation of $R, G$ a nonzero generalized derivation of $R$, and $f\left(x_{1}, \ldots, x_{n}\right)$ a non-central multilinear polynomial over $C$ such that $\delta(G(f(X)) f(X))=0$ for all $X=\left(x_{1}, \ldots, x_{n}\right) \in R^{n}$, then there exist $a, b \in U$ such that $G(x)=a x$ and $\delta(x)=[b, x]$ for all $x \in R$, with $[b, a]=0$ and $f\left(x_{1}, \ldots, x_{n}\right)^{2}$ is central-valued on $R$.

Further, the first author and Argac 8 extended the above result replacing derivation $\delta$ with another generalized derivation $F$, that is, $F(G(f(X)) f(X))=0$ for all $X=$ $\left(x_{1}, \ldots, x_{n}\right) \in I^{n}$, and then gave the complete description of the additive maps $F$ and $G$, where $I$ is a non-zero two-sided ideal of $R$.

In another paper [1], Argaç and De Filippis studied the generalized derivations $G$ and $H$ co-commuting on $f(I)=\left\{f\left(x_{1}, \ldots, x_{n}\right) \mid x_{i} \in I\right\}$, that is, $G(u) u-u H(u)=0$ for all $u \in f(I)$ and then obtained the all possible forms of the maps $F$ and $G$, where $I$ is a non-zero two-sided ideal of $R$.

Motivated by the above results we prove the following theorem.

Theorem 1.1. Let $R$ be a noncommutative prime ring of characteristic different from 2 with Utumi quotient ring $U$ and extended centroid $C$, and $f\left(x_{1}, \ldots, x_{n}\right)$ a multilinear polynomial over $C$, which is not central valued on $R$. Suppose that $F, G$ and $H$ are three 
nonzero generalized derivations of $R$ such that

$$
F(G(f(X)) f(X))=f(X) H(f(X))
$$

for all $X=\left(x_{1}, \ldots, x_{n}\right) \in R^{n}$. Then one of the following holds:

(1) there exist $\lambda \in C$ and $a, b \in U$ such that $F(x)=\lambda x, G(x)=x a$ and $H(x)=\lambda a x$ for all $x \in R$;

(2) there exist $\lambda, \alpha \in C$ and $p, q, u, v \in U$ such that $F(x)=\lambda x, G(x)=p x+x q$ and $H(x)=\lambda(q x+x p)$ for all $x \in R$ with $f\left(x_{1}, \ldots, x_{n}\right)^{2}$ is central valued in $R$;

(3) there exist $\lambda \in C$ and $a, p \in U$ such that $F(x)=a x, G(x)=p x$ and $H(x)=\lambda x$ for all $x \in R$ with ap $=\lambda$;

(4) there exist $\lambda \in C$ and $a \in U$ such that $F(x)=x a, G(x)=\lambda x$ and $H(x)=\lambda x a$ for all $x \in R$;

(5) there exist $a, b, p, v \in U$ such that $F(x)=a x+x b, G(x)=p x$ and $H(x)=x v$ for all $x \in R$ with $f\left(x_{1}, \ldots, x_{n}\right)^{2}$ is central valued on $R$ and $a p+p b=v$.

In particular, when $G=H$, we have the following

Corollary 1.2. Let $R$ be a noncommutative prime ring of characteristic different from 2 with Utumi quotient ring $U$ and extended centroid $C$, and $f\left(x_{1}, \ldots, x_{n}\right)$ a multilinear polynomial over $C$, which is not central valued on $R$. Suppose that $F$ and $G$ are two nonzero generalized derivations of $R$ such that

$$
F(G(f(X)) f(X))=f(X) G(f(X))
$$

for all $X=\left(x_{1}, \ldots, x_{n}\right) \in R^{n}$. Then one of the following holds:

(1) there exists $\mu \in C$ such that $F(x)=x$ and $G(x)=\mu x$ for all $x \in R$;

(2) there exist $\alpha \in C$ and $p \in U$ such that $F(x)=x$ and $G(x)=p x+x p+\alpha x$ for all $x \in R$ with $f\left(x_{1}, \ldots, x_{n}\right)^{2}$ is central valued in $R$;

(3) there exists $p \in U$ such that $F(x)=-x$ and $G(x)=[p, x]$ for all $x \in R$ with $f\left(x_{1}, \ldots, x_{n}\right)^{2}$ is central valued in $R$;

(4) there exist $a, b, p \in U$ such that $F(x)=a x+x b$ and $G(x)=p x$ for all $x \in R$ with $f\left(x_{1}, \ldots, x_{n}\right)^{2}$ is central valued on $R$ and $F(p)=p$. 
Proof. By Theorem 1.1, we have the following conclusions:

(1) There exists $\mu \in C$ such that $F(x)=x$ and $G(x)=\mu x$ for all $x \in R$. This is our conclusion (1).

(2) There exist $\lambda, \alpha \in C$ and $p, q \in U$ such that $F(x)=\lambda x, G(x)=p x+x q$ for all $x \in R$ with $f\left(x_{1}, \ldots, x_{n}\right)^{2}$ being central valued in $R$ and $q-\lambda p=\lambda q-p=\alpha \in C$. The last relation yields $(\lambda-1)(p+q)=0$. This yields either $\lambda=1$ or $p+q=0$. (i) When $\lambda=1$, we have $q-p=\alpha \in C$ and hence $F(x)=x$ and $G(x)=p x+x p+\alpha x$ for all $x \in R$. This gives conclusion (2). (ii) When $p+q=0$, we have $F(x)=\lambda x, G(x)=[p, x]$ for all $x \in R$ with $p+\lambda p=\alpha \in C$, i.e., $(1+\lambda) p \in C$. This implies $1+\lambda=0$, since $p \in C$ implies $G=0$, a contradiction. Thus $\lambda=-1$. This gives conclusion (3).

(3) There exist $\lambda \in C$ and $a, p \in U$ such that $F(x)=a x, G(x)=\lambda x$ for all $x \in R$ with $a \lambda=\lambda$. Since $G \neq 0, \lambda \neq 0$ and hence last relation gives $a=1$. This is conclusion (1).

(4) There exist $\lambda \in C$ and $a, u \in U$ such that $F(x)=x a, G(x)=\lambda x$ for all $x \in R$ with $a \lambda=\lambda$. By the same argument as above, $a=1$, as desired in (1).

(5) There exist $a, b, p, v \in U$ such that $F(x)=a x+x b, G(x)=p x$ for all $x \in R$ with $f\left(x_{1}, \ldots, x_{n}\right)^{2}$ being central valued on $R$ and $a p+p b=p$. This is conclusion (4).

From Theorem 1.1 (2), we conclude that when $G$ is derivation then $H$ also be a derivation. Thus following corollary is straightforward.

Corollary 1.3. Let $R$ be a noncommutative prime ring of characteristic different from 2 with Utumi quotient ring $U$ and extended centroid $C$, and $f\left(x_{1}, \ldots, x_{n}\right)$ a multilinear polynomial over $C$, which is not central valued on $R$. Suppose that $F$ and $H$ are two nonzero generalized derivations of $R$ and $d$ is a derivation of $R$ such that

$$
F(d(f(X)) f(X))=f(X) H(f(X))
$$

for all $X=\left(x_{1}, \ldots, x_{n}\right) \in R^{n}$. Then there exist $\lambda \in C$ and $p, u \in U$ such that $F(x)=\lambda x$, $d(x)=[p, x]$ and $H(x)=-\lambda[p, x]$ for all $x \in R$ with $f\left(x_{1}, \ldots, x_{n}\right)^{2}$ being central valued in $R$.

Corollary 1.4. Let $R$ be a noncommutative prime ring of characteristic different from 2 with Utumi quotient ring $U$ and extended centroid $C$, and $f\left(x_{1}, \ldots, x_{n}\right)$ a multilinear polynomial over $C$, which is not central valued on $R$. Suppose that $F$ and $G$ are two nonzero generalized derivations of $R$ and $d$ is a derivation of $R$ such that

$$
F(G(f(X)) f(X))=f(X) d(f(X))
$$

for all $X=\left(x_{1}, \ldots, x_{n}\right) \in R^{n}$. Then there exist $\lambda \in C$ and $p, u \in U$ such that $F(x)=\lambda x$, $G(x)=[p, x]$ and $d(x)=-\lambda[p, x]$ for all $x \in R$ with $f\left(x_{1}, \ldots, x_{n}\right)^{2}$ being central valued in $R$. 
In particular, when $F$ is derivation, then we have last conclusion of Theorem 1.1 .

Corollary 1.5. Let $R$ be a noncommutative prime ring of characteristic different from 2 with Utumi quotient ring $U$ and extended centroid $C$, and $f\left(x_{1}, \ldots, x_{n}\right)$ a multilinear polynomial over $C$, which is not central valued on $R$. Suppose that $G$ and $H$ are two nonzero generalized derivations of $R$ and $d$ is a derivation of $R$ such that

$$
d(G(f(X)) f(X))=f(X) H(f(X))
$$

for all $X=\left(x_{1}, \ldots, x_{n}\right) \in R^{n}$. Then there exist $a, b, p, v \in U$ such that $d(x)=[a, x]$, $G(x)=p x$ and $H(x)=x v$ for all $x \in R$ with $f\left(x_{1}, \ldots, x_{n}\right)^{2}$ being central valued on $R$ and $d(p)=v$.

Corollary 1.6. Let $R$ be a noncommutative prime ring of characteristic different from 2 with Utumi quotient ring $U$ and extended centroid $C$, and $f\left(x_{1}, \ldots, x_{n}\right)$ a multilinear polynomial over $C$. Suppose that $d, \delta$ and $h$ are three nonzero derivations of $R$ such that

$$
d(\delta(f(X)) f(X))=f(X) h(f(X))
$$

for all $X=\left(x_{1}, \ldots, x_{n}\right) \in R^{n}$. Then $f\left(x_{1}, \ldots, x_{n}\right)$ must be central valued.

Corollary 1.7. Let $R$ be a prime ring of characteristic different from 2. Suppose that d, $\delta$ and $h$ are three nonzero derivations of $R$ such that

$$
d(\delta(x) x)=x h(x)
$$

for all $x \in R$. Then $R$ must be commutative.

\section{Main results}

Let $F(\neq 0), G(\neq 0)$ and $H(\neq 0)$ be all inner generalized derivations of $R$. There exist some fixed $a, b, p, q, u, v \in U$ such that $F(x)=a x+x b, G(x)=p x+x q$ and $H(x)=u x+x v$ for all $x \in R$. Then by our hypothesis $F(G(x) x)=x H(x))$ for all $x \in f(R)$, we have

$$
a p x^{2}+a x q x+p x^{2} b+x q x b-x u x-x^{2} v=0
$$

for all $x \in f(R)$.

To investigate this generalized polynomial identity (GPI) in prime ring $R$, we recall the following

Lemma 2.1. [1, Lemma 3] Let $R$ be a noncommutative prime ring with Utumi quotient ring $U$ and extended centroid $C$, and $f\left(x_{1}, \ldots, x_{n}\right)$ a multilinear polynomial over $C$, which is not central valued on $R$. Suppose that there exist $a, b, c, q \in U$ such that $(a f(r)+$ $f(r) b) f(r)-f(r)(c f(r)+f(r) q)=0$ for all $r=\left(r_{1}, \ldots, r_{n}\right) \in R^{n}$. Then one of the following holds: 
(1) $a, q \in C$ and $q-a=b-c=\alpha \in C$;

(2) $f\left(x_{1}, \ldots, x_{n}\right)^{2}$ is central valued on $R$ and there exists $\alpha \in C$ such that $q-a=b-c=$ $\alpha$;

(3) $\operatorname{char}(R)=2$ and $R$ satisfies $s_{4}$.

Now to investigate our generalized polynomial identity (GPI) (2.1), in all that follows, we assume $R$ a noncommutative prime ring with extended centroid $C, \operatorname{char}(R) \neq 2$. Moreover, we assume that $f\left(x_{1}, \ldots, x_{n}\right)$ is a multilinear polynomial over $C$ which is not central valued on $R$.

Lemma 2.2. If $a, b \in C$ and $R$ satisfies (2.1), then one of the following holds:

(1) $p, v \in C$ with $(a+b)(p+q)=u+v$;

(2) $f\left(x_{1}, \ldots, x_{n}\right)^{2}$ is central valued in $R$ with $v-(a+b) p=(a+b) q-u=\alpha \in C$.

Proof. If $a, b \in C$, then by hypothesis

$$
(a+b)(p x+x q) x=x(u x+x v)
$$

for all $x \in f(R)$. In this case by Lemma 2.1, one of the following holds:

(i) $(a+b) p, v \in C$ and $v-(a+b) p=(a+b) q-u=\alpha \in C$. Since $F \neq 0, a+b \neq 0$ and so $(a+b) p \in C$ implies $p \in C$.

(ii) $f\left(x_{1}, \ldots, x_{n}\right)^{2}$ is central valued on $R$ and there exists $\alpha \in C$ such that $v-(a+b) p=$ $(a+b) q-u=\alpha \in C$.

Lemma 2.3. If $q \in C$ and $R$ satisfies (2.1), then one of the following holds:

(1) $b, u, v \in C$ with $(a+b)(p+q)=v+u$;

(2) $a, p, u \in C$ with $(a+b)(p+q)=v+u$;

(3) $u \in C$ with $f\left(x_{1}, \ldots, x_{n}\right)^{2}$ being central valued on $R$ and $a(p+q)+(p+q) b=u+v$.

Proof. If $q \in C$, then our hypothesis becomes

$$
a(p+q) x^{2}+p x^{2} b+x^{2}(b q-v)-x u x=0
$$

for all $x \in f(R)$. Then by Proposition 2.7 in [10], we conclude that $u \in C$. Then our hypothesis reduces to

$$
a(p+q) x^{2}+p x^{2} b+x^{2}(b q-v-u)=0
$$

for all $x \in f(R)$. Then by applying Lemma 2.9 in [7], we conclude one of the following: 
(i) $b, u, b q-v-u \in C$ with $a(p+q)+p b+(b q-v-u)=0$, i.e., $(a+b)(p+q)=v+u$. Since $b, q, u \in C$, we have $v \in C$.

(ii) $a(p+q), u, p \in C$ with $a(p+q)+p b+(b q-v-u)=0$, i.e., $(a+b)(p+q)=v+u$. In this case $G(x)=(p+q) x$ for all $x \in R$. As $G \neq 0$, thus $0 \neq p+q \in C$. Hence $a(p+q) \in C$ implies $a \in C$.

(iii) $u \in C$ and $f\left(x_{1}, \ldots, x_{n}\right)^{2}$ is central valued on $R$ with $a(p+q)+p b+(b q-v-u)=0$, i.e., $a(p+q)+(p+q) b=u+v$.

Thus the lemma is proved.

Lemma 2.4. Let $R$ be a prime ring with extended centroid $C$ and $a, b, p, q, u, v \in R$. If

$$
a p x^{2}+a x q x+p x^{2} b+x q x b-x u x-x^{2} v=0
$$

for all $x \in f(R)$ is a trivial generalized polynomial identity, then either $a, b \in C$ or $q \in C$.

Proof. Let $a \notin C$ and $q \notin C$. By hypothesis, we have

$$
\begin{aligned}
\zeta\left(x_{1}, \ldots, x_{n}\right)= & \operatorname{apf}\left(x_{1}, \ldots, x_{n}\right)^{2}+a f\left(x_{1}, \ldots, x_{n}\right) q f\left(x_{1}, \ldots, x_{n}\right) \\
& +p f\left(x_{1}, \ldots, x_{n}\right)^{2} b+f\left(x_{1}, \ldots, x_{n}\right) q f\left(x_{1}, \ldots, x_{n}\right) b \\
& -f\left(x_{1}, \ldots, x_{n}\right) u f\left(x_{1}, \ldots, x_{n}\right)-f\left(x_{1}, \ldots, x_{n}\right)^{2} v=0
\end{aligned}
$$

for all $x_{1}, \ldots, x_{n} \in R$. Since $R$ and $U$ satisfy the same generalized polynomial identity (see [4]), $U$ satisfies $\zeta\left(x_{1}, \ldots, x_{n}\right)=0$. By our assumption $\zeta\left(x_{1}, \ldots, x_{n}\right)$ is a trivial GPI for $U$. Let $T=U *_{C} C\left\{x_{1}, x_{2}, \ldots, x_{n}\right\}$, the free product of $U$ and $C\left\{x_{1}, \ldots, x_{n}\right\}$, the free $C$-algebra in noncommuting indeterminates $x_{1}, x_{2}, \ldots, x_{n}$. Then, $\zeta\left(x_{1}, \ldots, x_{n}\right)$ is zero element in $T=U *_{C} C\left\{x_{1}, \ldots, x_{n}\right\}$. This implies that $\{a p, a, p, 1\}$ is linearly $C$-dependent. Then there exist $\alpha_{1}, \alpha_{2}, \alpha_{3}, \alpha_{4} \in C$ such that $\alpha_{1} a p+\alpha_{2} a+\alpha_{3} p+\alpha_{4} \cdot 1=0$. If $\alpha_{1}=\alpha_{3}=0$, then $\alpha_{2} \neq 0$ and so $a=-\alpha_{2}^{-1} \alpha_{4} \in C$, a contradiction. Therefore, either $\alpha_{1} \neq 0$ or $\alpha_{3} \neq 0$. Without loss of generality, we assume that $\alpha_{1} \neq 0$. Then $a p=\alpha a+\beta p+\gamma$, where $\alpha=-\alpha_{1}^{-1} \alpha_{2}, \beta=-\alpha_{1}^{-1} \alpha_{3}, \gamma=-\alpha_{1}^{-1} \alpha_{4}$. Then

$$
\begin{aligned}
& (\alpha a+\beta p+\gamma) f\left(x_{1}, \ldots, x_{n}\right)^{2}+a f\left(x_{1}, \ldots, x_{n}\right) q f\left(x_{1}, \ldots, x_{n}\right) \\
+ & p f\left(x_{1}, \ldots, x_{n}\right)^{2} b+f\left(x_{1}, \ldots, x_{n}\right) q f\left(x_{1}, \ldots, x_{n}\right) b \\
- & f\left(x_{1}, \ldots, x_{n}\right) u f\left(x_{1}, \ldots, x_{n}\right)-f\left(x_{1}, \ldots, x_{n}\right)^{2} v=0
\end{aligned}
$$

in $T$. This implies that $\{a, p, 1\}$ is linearly $C$-dependent. Then there exist $\beta_{1}, \beta_{2}, \beta_{3} \in C$ such that $\beta_{1} a+\beta_{2} p+\beta_{3}=0$. By same argument as before, since $a \notin C$, we have $\beta_{2} \neq 0$ and hence $p=\alpha^{\prime} a+\beta^{\prime}$ for some $\alpha^{\prime}, \beta^{\prime} \in C$. Thus our identity becomes

$$
\begin{aligned}
& \left(\alpha a+\beta \alpha^{\prime} a+\beta \beta^{\prime}+\gamma\right) f\left(x_{1}, \ldots, x_{n}\right)^{2}+a f\left(x_{1}, \ldots, x_{n}\right) q f\left(x_{1}, \ldots, x_{n}\right) \\
+ & \left(\alpha^{\prime} a+\beta^{\prime}\right) f\left(x_{1}, \ldots, x_{n}\right)^{2} b+f\left(x_{1}, \ldots, x_{n}\right) q f\left(x_{1}, \ldots, x_{n}\right) b \\
- & f\left(x_{1}, \ldots, x_{n}\right) u f\left(x_{1}, \ldots, x_{n}\right)-f\left(x_{1}, \ldots, x_{n}\right)^{2} v=0 .
\end{aligned}
$$


Since $\{a, 1\}$ is linearly $C$-independent, we have

$$
\left(\alpha+\beta \alpha^{\prime}\right) a f\left(x_{1}, \ldots, x_{n}\right)^{2}+a f\left(x_{1}, \ldots, x_{n}\right) q f\left(x_{1}, \ldots, x_{n}\right)+\alpha^{\prime} a f\left(x_{1}, \ldots, x_{n}\right)^{2} b=0,
$$

that is

$$
a f\left(x_{1}, \ldots, x_{n}\right)\left(\left(\alpha+\beta \alpha^{\prime}+q\right) f\left(x_{1}, \ldots, x_{n}\right)+\alpha^{\prime} f\left(x_{1}, \ldots, x_{n}\right) b\right)=0
$$

in $T$. Moreover, since $q \notin C$, the term af $\left(x_{1}, \ldots, x_{n}\right) q f\left(x_{1}, \ldots, x_{n}\right)$ cannot be canceled and hence $a f\left(x_{1}, \ldots, x_{n}\right) q f\left(x_{1}, \ldots, x_{n}\right)=0$ in $T$ which implies $q=0$, a contradiction. Thus either $a \in C$ or $q \in C$.

Similarly, we can prove that either $b \in C$ or $q \in C$.

Lemma 2.5. 6, Lemma 1] Let $K$ be an infinite field and $m \geq 2$. If $A_{1}, \ldots, A_{k}$ are not scalar matrices in $M_{m}(K)$ then there exists some invertible matrix $P \in M_{m}(K)$ such that any matrices $P A_{1} P^{-1}, \ldots, P A_{k} P^{-1}$ have all non-zero entries.

Proposition 2.6. Let $R=M_{m}(C)$ be the ring of all $m \times m$ matrices over the infinite field $C$ and $f\left(x_{1}, \ldots, x_{n}\right)$ a non-central multilinear polynomial over $C$. If there exist $a, b, p, q, u, v \in R$ such that

$$
a p x^{2}+a x q x+p x^{2} b+x q x b-x u x-x^{2} v=0
$$

for all $x \in f(R)$, then either $a, b$ are central or $q$ is central.

Proof. By our hypothesis, $R$ satisfies the generalized polynomial identity

$$
\begin{aligned}
& \operatorname{apf}\left(x_{1}, \ldots, x_{n}\right)^{2}+a f\left(x_{1}, \ldots, x_{n}\right) q f\left(x_{1}, \ldots, x_{n}\right) \\
+ & p f\left(x_{1}, \ldots, x_{n}\right)^{2} b+f\left(x_{1}, \ldots, x_{n}\right) q f\left(x_{1}, \ldots, x_{n}\right) b \\
- & f\left(x_{1}, \ldots, x_{n}\right) u f\left(x_{1}, \ldots, x_{n}\right)-f\left(x_{1}, \ldots, x_{n}\right)^{2} v=0 .
\end{aligned}
$$

We assume first that $a \notin Z(R)$ and $q \notin Z(R)$. Now we shall show that this case leads to a contradiction.

Since $a \notin Z(R)$ and $q \notin Z(R)$, by Lemma 2.5 there exists a $C$-automorphism $\phi$ of $M_{m}(C)$ such that $\phi(a), \phi(q)$ have all non-zero entries. Clearly, $R$ satisfies the generalized polynomial identity

$$
\begin{aligned}
& \phi(a p) f\left(x_{1}, \ldots, x_{n}\right)^{2}+\phi(a) f\left(x_{1}, \ldots, x_{n}\right) \phi(q) f\left(x_{1}, \ldots, x_{n}\right) \\
+ & \phi(p) f\left(x_{1}, \ldots, x_{n}\right)^{2} \phi(b)+f\left(x_{1}, \ldots, x_{n}\right) \phi(q) f\left(x_{1}, \ldots, x_{n}\right) b \\
- & f\left(x_{1}, \ldots, x_{n}\right) \phi(u) f\left(x_{1}, \ldots, x_{n}\right)-f\left(x_{1}, \ldots, x_{n}\right)^{2} \phi(v)=0 .
\end{aligned}
$$

By $e_{i j}$, we mean the usual matrix unit with 1 in $(i, j)$-entry and zero elsewhere. Since $f\left(x_{1}, \ldots, x_{n}\right)$ is not central valued, by [15] (see also [17]), there exist a sequence of matrices 
$v_{1}, \ldots, v_{n}$ in $M_{m}(C)$ and $\gamma \in C-\{0\}$ such that $f\left(v_{1}, \ldots, v_{n}\right)=\gamma e_{p q}$, with $p \neq q$. Moreover, since the set $\left\{f\left(r_{1}, \ldots, r_{n}\right): r_{1}, \ldots, r_{n} \in M_{m}(C)\right\}$ is invariant under the action of all $C$ automorphisms of $M_{m}(C)$, then for any $i \neq j$ there exist $r_{1}, \ldots, r_{n}$ in $M_{m}(C)$ such that $f\left(r_{1}, \ldots, r_{n}\right)=e_{i j}$. Hence by 2.2 , we have

$$
\phi(a) e_{i j} \phi(q) e_{i j}+e_{i j} \phi(q) e_{i j} b-e_{i j} \phi(u) e_{i j}=0
$$

and then left multiplying by $e_{i j}$, it follows $e_{i j} \phi(a) e_{i j} \phi(q) e_{i j}=0$, which is a contradiction, since $\phi(a)$ and $\phi(q)$ have all non-zero entries. Thus we conclude that either $a \in Z(R)$ or $q \in Z(R)$.

If we consider $b \notin Z(R)$ and $q \notin Z(R)$, then by same argument as above we have a contradiction with the fact $e_{i j} \phi(q) e_{i j} \phi(b) e_{i j}=0$ obtained from (2.3). Thus we conclude either $b \in Z(R)$ or $q \in Z(R)$.

Thus, $q \notin Z(R)$ implies $a \in Z(R)$ and $b \in Z(R)$. Thus the conclusion follows.

Proposition 2.7. Let $R=M_{m}(C)$ be the ring of all matrices over the field $C$ with $\operatorname{char}(R) \neq 2$ and $f\left(x_{1}, \ldots, x_{n}\right)$ a non-central multilinear polynomial over $C$. If there exist $a, b, p, q, u, v \in R$ such that

$$
a p x^{2}+a x q x+p x^{2} b+x q x b-x u x-x^{2} v=0
$$

for all $x \in f(R)$, then either $a, b \in C \cdot I_{m}$ or $q \in C \cdot I_{m}$.

Proof. In case $C$ is infinite, the conclusions follow by Proposition 2.6 .

So we assume that $C$ is finite. Let $K$ be an infinite field which is an extension of the field $C$. Let $\bar{R}=M_{m}(K) \cong R \otimes_{C} K$. Notice that the multilinear polynomial $f\left(x_{1}, \ldots, x_{n}\right)$ is central-valued on $R$ if and only if it is central-valued on $\bar{R}$. Consider the generalized polynomial

$$
\begin{aligned}
\Psi\left(x_{1}, \ldots, x_{n}\right)= & \operatorname{apf}\left(x_{1}, \ldots, x_{n}\right)^{2}+a f\left(x_{1}, \ldots, x_{n}\right) q f\left(x_{1}, \ldots, x_{n}\right) \\
& +p f\left(x_{1}, \ldots, x_{n}\right)^{2} b+f\left(x_{1}, \ldots, x_{n}\right) q f\left(x_{1}, \ldots, x_{n}\right) b \\
& -f\left(x_{1}, \ldots, x_{n}\right) u f\left(x_{1}, \ldots, x_{n}\right)-f\left(x_{1}, \ldots, x_{n}\right)^{2}
\end{aligned}
$$

which is a generalized polynomial identity for $R$.

Moreover, it is a multi-homogeneous of multi-degree $(2, \ldots, 2)$ in the indeterminates $x_{1}, \ldots, x_{n}$. Hence the complete linearization of $\Psi\left(x_{1}, \ldots, x_{n}\right)$ yields a multilinear generalized polynomial $\Theta\left(x_{1}, \ldots, x_{n}, y_{1}, \ldots, y_{n}\right)$ in $2 n$ indeterminates, moreover

$$
\Theta\left(x_{1}, \ldots, x_{n}, x_{1}, \ldots, x_{n}\right)=2^{n} \Psi\left(x_{1}, \ldots, x_{n}\right) .
$$

Clearly the multilinear polynomial $\Theta\left(x_{1}, \ldots, x_{n}, y_{1}, \ldots, y_{n}\right)$ is a generalized polynomial identity for $R$ and $\bar{R}$ too. Since $\operatorname{char}(C) \neq 2$ we obtain $\Psi\left(r_{1}, \ldots, r_{n}\right)=0$ for all $r_{1}, \ldots, r_{n} \in$ $\bar{R}$ and then conclusion follows from Proposition 2.6 . 
In particular, we have the following

Corollary 2.8. Let $R=M_{m}(C)$ be the ring of all matrices over the field $C$ with $\operatorname{char}(R) \neq$ 2. If there exist $a, b, p, q, u, v \in R$ such that

$$
a p x^{2}+a x q x+p x^{2} b+x q x b-x u x-x^{2} v=0
$$

for all $x \in R$, then either $a, b \in C \cdot I_{m}$ or $q \in C \cdot I_{m}$.

Similarly, we have the following

Corollary 2.9. Let $R=M_{m}(C)$ be the ring of all matrices over the field $C$ with $\operatorname{char}(R) \neq$ 2. If there exist $a^{\prime}, a, b, p, q, u, v \in R$ such that

$$
a^{\prime} x^{2}+a x q x+p x^{2} b+x q x b-x u x-x^{2} v=0
$$

for all $x \in R$, then either $a, b \in C \cdot I_{m}$ or $q \in C \cdot I_{m}$.

Lemma 2.10. Let $R$ be a primitive ring of $\operatorname{char}(R) \neq 2$ with nonzero socle $\operatorname{Soc}(R)$, which is isomorphic to a dense ring of linear transformations of a vector space $V$ over $C$, such that $\operatorname{dim}_{C} V=\infty$. Let $a^{\prime}, a, b, p, q, u, v \in R$. If

$$
a^{\prime} x^{2}+a x q x+p x^{2} b+x q x b-x u x-x^{2} v=0
$$

for all $x \in R$, then either $a, b \in C$ or $q \in C$.

Proof. Recall that if any element $r \in R$ commutes the nonzero ideal $\operatorname{Soc}(R C)$, i.e., $[r, \operatorname{Soc}(R C)]=(0)$, then $r \in C$. Hence on contrary, we assume that there exist $h_{0}, h_{1}, h_{2} \in$ $\operatorname{Soc}(R)$ such that

(i) either $\left[a, h_{0}\right] \neq 0$ or $\left[b, h_{1}\right] \neq 0$;

(ii) $\left[q, h_{2}\right] \neq 0$

and prove that a number of contradiction arises. Since $V$ is infinite dimensional over $C$, for any $e=e^{2} \in \operatorname{Soc}(R)$, we have $e R e \cong M_{k}(C)$ with $k=\operatorname{dim}_{C} V e$. By Litoff's Theorem [12], there exists an idempotent $e \in \operatorname{Soc}(R)$ such that

- $h_{0}, h_{1}, h_{2} \in e R e$;

- $h_{0} a, a h_{0}, h_{1} a, a h_{1}, h_{2} a, a h_{2} \in e R e$;

- $h_{0} b, b h_{0}, h_{1} b, b h_{1}, h_{2} b, b h_{2} \in e R e ;$

- $h_{0} q, q h_{0}, h_{1} q, q h_{1}, h_{2} q, q h_{2} \in e R e$, 
where $e R e \cong M_{k}(C), k=\operatorname{dim}_{C} V e$. Since $R$ satisfies $e\left\{a^{\prime}(\text { exe })^{2}+\right.$ aexeqexe $+p(e x e)^{2} b+$ exeqexeb-exeuexe $\left.-(e x e)^{2} v\right\} e=0$, the subring eRe satisfies $e a^{\prime} e x^{2}+$ eaexeqex + epe $x^{2}$ ebe $+x e q e x e b e-x e u e x-x^{2}$ eve $=0$. By Corollary 2.9. we conclude that one of the following holds:

(i) eae, ebe $\in e C$ which contradicts with the choice of $h_{0}$ and $h_{1}$;

(ii) eqe $\in e C$ which contradicts with the choices of $h_{2}$.

Lemma 2.11. Let $R$ be a noncommutative prime ring of characteristic different from 2 with Utumi quotient ring $U$ and extended centroid $C$, and $f\left(x_{1}, \ldots, x_{n}\right)$ a multilinear polynomial over $C$, which is not central valued on $R$. Suppose that $F, G$ and $H$ are three nonzero inner generalized derivations of $R$ such that $F(G(f(r)) f(r))=f(r) H(f(r))$ for all $r=\left(r_{1}, \ldots, r_{n}\right) \in R^{n}$, then one of the following holds:

(1) there exist $\lambda \in C$ and $a, b \in U$ such that $F(x)=\lambda x, G(x)=x a$ and $H(x)=b x$ for all $x \in R$ with $\lambda a=b$;

(2) there exist $\lambda, \alpha \in C$ and $p, q, u, v \in U$ such that $F(x)=\lambda x, G(x)=p x+x q$ and $H(x)=u x+x v$ for all $x \in R$ with $f\left(x_{1}, \ldots, x_{n}\right)^{2}$ being central valued in $R$ and $v-\lambda p=\lambda q-u=\alpha \in C$;

(3) there exist $\lambda \in C$ and $a, p \in U$ such that $F(x)=a x, G(x)=p x$ and $H(x)=\lambda x$ for all $x \in R$ with ap $=\lambda$;

(4) there exist $\lambda \in C$ and $a, u \in U$ such that $F(x)=x a, G(x)=\lambda x$ and $H(x)=x u$ for all $x \in R$ with $a \lambda=u$;

(5) there exist $a, b, p, v \in U$ such that $F(x)=a x+x b, G(x)=p x$ and $H(x)=x v$ for all $x \in R$ with $f\left(x_{1}, \ldots, x_{n}\right)^{2}$ being central valued on $R$ and $a p+p b=v$.

Proof. Suppose that for some $a, b, p, q, u, v \in U, F(x)=a x+x b, G(x)=p x+x q$ and $H(x)=u x+x v$ for all $x \in R$. By hypothesis, we have

$$
\begin{aligned}
& a\left(\left(p f\left(x_{1}, \ldots, x_{n}\right)+f\left(x_{1}, \ldots, x_{n}\right) q\right) f\left(x_{1}, \ldots, x_{n}\right)\right) \\
& +\left(\left(p f\left(x_{1}, \ldots, x_{n}\right)+f\left(x_{1}, \ldots, x_{n}\right) q\right) f\left(x_{1}, \ldots, x_{n}\right)\right) b \\
= & f\left(x_{1}, \ldots, x_{n}\right)\left(u f\left(x_{1}, \ldots, x_{n}\right)+f\left(x_{1}, \ldots, x_{n}\right) v\right),
\end{aligned}
$$

that is,

$$
\begin{aligned}
& \operatorname{apf}\left(x_{1}, \ldots, x_{n}\right)^{2}+a f\left(x_{1}, \ldots, x_{n}\right) q f\left(x_{1}, \ldots, x_{n}\right) \\
+ & p f\left(x_{1}, \ldots, x_{n}\right)^{2} b+f\left(x_{1}, \ldots, x_{n}\right) q f\left(x_{1}, \ldots, x_{n}\right) b \\
- & f\left(x_{1}, \ldots, x_{n}\right) u f\left(x_{1}, \ldots, x_{n}\right)-f\left(x_{1}, \ldots, x_{n}\right)^{2} v=0
\end{aligned}
$$


for all $x_{1}, \ldots, x_{n} \in R$. Since $R$ and $U$ satisfy the same generalized polynomial identities (see [4]), therefore, $U$ satisfies

$$
\begin{aligned}
& \operatorname{apf}\left(x_{1}, \ldots, x_{n}\right)^{2}+a f\left(x_{1}, \ldots, x_{n}\right) q f\left(x_{1}, \ldots, x_{n}\right) \\
+ & p f\left(x_{1}, \ldots, x_{n}\right)^{2} b+f\left(x_{1}, \ldots, x_{n}\right) q f\left(x_{1}, \ldots, x_{n}\right) b \\
- & f\left(x_{1}, \ldots, x_{n}\right) u f\left(x_{1}, \ldots, x_{n}\right)-f\left(x_{1}, \ldots, x_{n}\right)^{2} v=0 .
\end{aligned}
$$

If this is a trivial generalized polynomial identity for $U$, then by Lemma 2.4, either $a, b \in C$ or $q \in C$.

Next we assume that (2.4) is a non-trivial GPI for $U$.

Since both $U$ and $U \otimes_{C} \bar{C}$ are prime and centrally closed [11, Theorems 2.5 and 3.5], we may replace $R$ by $U$ or $U \otimes_{C} \bar{C}$ according as $C$ finite or infinite. Then $R$ is centrally closed over $C$ and $R$ satisfies (2.4). By Martindale's Theorem [18], $R$ is then a primitive ring with nonzero socle $\operatorname{soc}(R)$ and with $C$ as its associated division ring. Then, by Jacobson's Theorem [13, p. 75], $R$ is isomorphic to a dense ring of linear transformations of a vector space $V$ over $C$. Assume first that $V$ is finite dimensional over $C$, that is, $\operatorname{dim}_{C} V=m$. By density of $R$, we have $R \cong M_{m}(C)$. Since $f\left(r_{1}, \ldots, r_{n}\right)$ is not central valued on $R$, $R$ must be noncommutative and so $m \geq 2$. In this case, by Proposition 2.7, we get that $a, b \in C$ or $q \in C$. If $V$ is infinite dimensional over $C$, then by Lemma 2.10, we conclude that either $a, b \in C$ or $q \in C$.

Thus up to now, we have proved that in any cases either $a, b \in C$ or $q \in C$.

Case 1: $a, b \in C$. In this case by Lemma 2.2, we have the following cases:

(i) $p, v \in C$ with $(a+b)(p+q)=u+v$; Thus $F(x)=a x+x b=(a+b) x, G(x)=$ $p x+x q=x(p+q)$ and $H(x)=u x+x v=(u+v) x$ for all $x \in R$. This is our conclusion (1).

(ii) $f\left(x_{1}, \ldots, x_{n}\right)^{2}$ is central valued in $R$ with $v-(a+b) p=(a+b) q-u=\alpha \in C$. Thus $F(x)=a x+x b=(a+b) x, G(x)=p x+x q$ and $H(x)=u x+x v$ for all $x \in R$. This is our conclusion $(2)$.

Case 2: $q \in C$. In this case by Lemma 2.3, we have the following cases:

(i) $b, q, u, v \in C$ with $(a+b)(p+q)=v+u=\lambda \in C$. Thus $F(x)=(a+b) x$, $G(x)=(p+q) x$ and $H(x)=(u+v) x$ for all $x \in R$. This is our conclusion (3).

(ii) $a, u, p, q \in C$ with $(a+b)(p+q)=v+u$. Thus $F(x)=x(a+b), G(x)=(p+q) x$ and $H(x)=x(u+v)$ for all $x \in R$. This is our conclusion (4).

(iii) $q, u \in C$ with $f\left(x_{1}, \ldots, x_{n}\right)^{2}$ being central valued on $R$ and $a(p+q)+(p+q) b=u+v$. Thus $F(x)=a x+x b, G(x)=(p+q) x$ and $H(x)=x(u+v)$ for all $x \in R$. This is our conclusion (5).

In particular we have 
Corollary 2.12. Let $R$ be a noncommutative prime ring of characteristic different from 2 with Utumi quotient ring $U$ and extended centroid $C$, and $f\left(x_{1}, \ldots, x_{n}\right)$ a multilinear polynomial over $C$, which is not central valued on $R$. Suppose that $F$ is a nonzero inner generalized derivation of $R$ such that $F([p, f(r)] f(r))=f(r)[q, f(r)]$ for all $r=\left(r_{1}, \ldots, r_{n}\right) \in$ $R^{n}$, then there exists $\lambda \in C$ such that $F(x)=\lambda x$ for all $x \in R$ with $f\left(x_{1}, \ldots, x_{n}\right)^{2}$ being central valued in $R$ and $(\lambda p+q) \in C$.

Corollary 2.13. Let $R$ be a noncommutative prime ring of characteristic different from 2 with Utumi quotient ring $U$ and extended centroid $C$, and $f\left(x_{1}, \ldots, x_{n}\right)$ a multilinear polynomial over $C$, which is not central valued on $R$. Suppose that $F$ is a nonzero inner generalized derivation of $R$ such that $F([p, f(r)] f(r))=f(r)[p, f(r)]$ for all $r=\left(r_{1}, \ldots, r_{n}\right) \in$ $R^{n}$, then there exists $\lambda \in C$ such that $F(x)=\lambda x$ for all $x \in R$ with $f\left(x_{1}, \ldots, x_{n}\right)^{2}$ being central valued in $R$ and $(\lambda+1) p \in C$.

Lemma 2.14. Let $R$ be a noncommutative prime ring of characteristic different from 2 with Utumi quotient ring $U$ and extended centroid $C$, and $f\left(x_{1}, \ldots, x_{n}\right)$ a multilinear polynomial over $C$, which is not central valued on $R$. Suppose that $G$ and $H$ are two generalized derivations of $R$ and $F(x)=c x+x c^{\prime}$ for all $x \in R$, for some $c, c^{\prime} \in U$ is a nonzero inner generalized derivation of $R$, such that $F(G(f(r)) f(r))=f(r) H(f(r))$ for all $r=\left(r_{1}, \ldots, r_{n}\right) \in R^{n}$, then one of the following holds:

(1) there exist $\lambda \in C$ and $a, b \in U$ such that $F(x)=\lambda x, G(x)=x a$ and $H(x)=b x$ for all $x \in R$ with $\lambda a=b$;

(2) there exist $\lambda, \alpha \in C$ and $p, q, u, v \in U$ such that $F(x)=\lambda x, G(x)=p x+x q$ and $H(x)=u x+x v$ for all $x \in R$ with $f\left(x_{1}, \ldots, x_{n}\right)^{2}$ being central valued in $R$ and $v-\lambda p=\lambda q-u=\alpha \in C$.

(3) there exist $\lambda \in C$ and $a, p \in U$ such that $F(x)=a x, G(x)=p x$ and $H(x)=\lambda x$ for all $x \in R$ with ap $=\lambda$.

(4) there exist $\lambda \in C$ and $a, u \in U$ such that $F(x)=x a, G(x)=\lambda x$ and $H(x)=x u$ for all $x \in R$ with $a \lambda=u$.

(5) there exist $a, b, p, v \in U$ such that $F(x)=a x+x b, G(x)=p x$ and $H(x)=x v$ for all $x \in R$ with $f\left(x_{1}, \ldots, x_{n}\right)^{2}$ being central valued on $R$ and $a p+p b=v$.

Proof. In view of [16. Theorem 3], we may assume that there exist $a, b \in U$ and derivations $d^{\prime}, \delta$ of $U$ such that $G(x)=a x+d^{\prime}(x)$ and $H(x)=b x+\delta(x)$. Since $R$ and $U$ satisfy the same generalized polynomial identities (see [4]) as well as the same differential identities (see 15]), we may assume that

$$
c\left\{a f(r)^{2}+d^{\prime}(f(r)) f(r)\right\}+\left\{a f(r)^{2}+d^{\prime}(f(r)) f(r)\right\} c^{\prime}=f(r) b f(r)+f(r) \delta(f(r))
$$


for all $r=\left(r_{1}, \ldots, r_{n}\right) \in U^{n}$, where $d^{\prime}, \delta$ are two derivations on $U$.

If $G$ and $H$ both are inner generalized derivations of $R$, then by Lemma 2.11 we obtain our conclusions (1)-(5). Thus we assume that not both of $F$ and $G$ are inner. Then $d^{\prime}$ and $\delta$ cannot be both inner derivations of $U$. Now we consider the following two cases:

Case I: Assume that $d^{\prime}$ and $\delta$ are $C$-dependent modulo inner derivations of $U$, say $\alpha d^{\prime}+\beta \delta=a d_{q}$, where $\alpha, \beta \in C, q \in U$ and $a d_{q}(x)=[q, x]$ for all $x \in R$.

Subcase $i$ : Let $\alpha \neq 0$. Then $d^{\prime}(x)=\lambda \delta(x)+[p, x]$ for all $x \in U$, for some $\lambda \in C$ and $p \in U$.

Then $\delta$ cannot be inner derivation of $U$. From $(2.5)$, we obtain

$$
\begin{aligned}
& c\left\{a f(r)^{2}+\lambda \delta(f(r)) f(r)+[p, f(r)] f(r)\right\} \\
& +\left\{a f(r)^{2}+\lambda \delta(f(r)) f(r)+[p, f(r)] f(r)\right\} c^{\prime} \\
= & f(r) b f(r)+f(r) \delta(f(r))
\end{aligned}
$$

for all $r=\left(r_{1}, \ldots, r_{n}\right) \in U^{n}$.

Since $f\left(r_{1}, \ldots, r_{n}\right)$ is a multilinear polynomial over $C$, we have $\delta\left(f\left(r_{1}, \ldots, r_{n}\right)\right)=$ $f^{\delta}\left(r_{1}, \ldots, r_{n}\right)+\sum_{i} f\left(r_{1}, \ldots, \delta\left(r_{i}\right), \ldots, r_{n}\right)$, where $f^{\delta}\left(r_{1}, \ldots, r_{n}\right)$ is the polynomials obtained from $f\left(r_{1}, \ldots, r_{n}\right)$ replacing each coefficients $\alpha_{\sigma}$ with $\delta\left(\alpha_{\sigma}\right)$. Thus by Kharchenko's Theorem [14, we can replace $\delta\left(f\left(r_{1}, \ldots, r_{n}\right)\right)$ by $f^{\delta}\left(r_{1}, \ldots, r_{n}\right)+\sum_{i} f\left(r_{1}, \ldots, y_{i}, \ldots, r_{n}\right)$ in (2.6) and then $U$ satisfies blended components

$$
\begin{aligned}
& c\left\{\lambda \sum_{i} f\left(r_{1}, \ldots, y_{i}, \ldots, r_{n}\right) f\left(r_{1}, \ldots, r_{n}\right)\right\} \\
& +\left\{\lambda \sum_{i} f\left(r_{1}, \ldots, y_{i}, \ldots, r_{n}\right) f\left(r_{1}, \ldots, r_{n}\right)\right\} c^{\prime} \\
& =f\left(r_{1}, \ldots, r_{n}\right) \sum_{i} f\left(r_{1}, \ldots, y_{i}, \ldots, r_{n}\right) .
\end{aligned}
$$

Replacing $y_{i}$ with $\left[q, y_{i}\right]$ for some $q \notin C$ in $(2.7)$, we obtain

$$
c \lambda[q, f(r)] f(r)+[q, f(r)] f(r) \lambda c^{\prime}=f(r)[q, f(r)] .
$$

By Corollary 2.13, $f\left(x_{1}, \ldots, x_{n}\right)^{2}$ is central valued in $R$ with $c \lambda, c^{\prime} \lambda \in C$ and $\left(\lambda\left(c+c^{\prime}\right)+\right.$ 1) $q \in C$. Since $q \notin C,\left(\lambda\left(c+c^{\prime}\right)+1\right) q \in C$ implies $\left(\lambda\left(c+c^{\prime}\right)+1\right)=0$, i.e., $\lambda\left(c+c^{\prime}\right)=-1$. Then by (2.7),

$$
\left(c+c^{\prime}\right) \lambda \sum_{i} f\left(r_{1}, \ldots, y_{i}, \ldots, r_{n}\right) f\left(r_{1}, \ldots, r_{n}\right)=f\left(r_{1}, \ldots, r_{n}\right) \sum_{i} f\left(r_{1}, \ldots, y_{i}, \ldots, r_{n}\right)
$$

which implies

$$
f\left(r_{1}, \ldots, r_{n}\right) \sum_{i} f\left(r_{1}, \ldots, y_{i}, \ldots, r_{n}\right)+\sum_{i} f\left(r_{1}, \ldots, y_{i}, \ldots, r_{n}\right) f\left(r_{1}, \ldots, r_{n}\right)=0 .
$$


In particular, for $y_{1}=r_{1}$ and $y_{2}=\cdots=y_{n}=0$, we have $2 f\left(r_{1}, \ldots, r_{n}\right)^{2}=0$ for all $r_{1}, \ldots, r_{n} \in U$, implying $f\left(r_{1}, \ldots, r_{n}\right)=0$ for all $r_{1}, \ldots, r_{n} \in U$, a contradiction.

Subcase ii: Let $\alpha=0$. Then $\delta(x)=\left[q^{\prime}, x\right]$ for all $x \in U$, where $q^{\prime}=\beta^{-1} q$. Since $\delta$ is inner, $d^{\prime}$ cannot be inner derivation. From $(2.5)$, we obtain

$$
\begin{aligned}
& c\left\{a f(r)^{2}+d^{\prime}(f(r)) f(r)\right\}+\left\{a f(r)^{2}+d^{\prime}(f(r)) f(r)\right\} c^{\prime} \\
= & f(r) b f(r)+f(r)\left[q^{\prime}, f(r)\right]
\end{aligned}
$$

for all $r=\left(r_{1}, \ldots, r_{n}\right) \in U^{n}$.

Since $d^{\prime}\left(f\left(r_{1}, \ldots, r_{n}\right)\right)=f^{d^{\prime}}\left(r_{1}, \ldots, r_{n}\right)+\sum_{i} f\left(r_{1}, \ldots, d^{\prime}\left(r_{i}\right), \ldots, r_{n}\right)$, by Kharchenko's Theorem 14, we can replace $d^{\prime}\left(f\left(r_{1}, \ldots, r_{n}\right)\right)$ by $f^{d^{\prime}}\left(r_{1}, \ldots, r_{n}\right)+\sum_{i} f\left(r_{1}, \ldots, y_{i}, \ldots, r_{n}\right)$ in (2.8) and then $U$ satisfies blended component

$$
\begin{aligned}
& c \sum_{i} f\left(r_{1}, \ldots, y_{i}, \ldots, r_{n}\right) f\left(r_{1}, \ldots, r_{n}\right) \\
+ & \sum_{i} f\left(r_{1}, \ldots, y_{i}, \ldots, r_{n}\right) f\left(r_{1}, \ldots, r_{n}\right) c^{\prime}=0 .
\end{aligned}
$$

Replacing $y_{i}$ with $\left[a^{\prime}, r_{i}\right]$ for some $a^{\prime} \notin C, U$ satisfies

$$
c\left[a^{\prime}, f\left(r_{1}, \ldots, r_{n}\right)\right] f\left(r_{1}, \ldots, r_{n}\right)+\left[a^{\prime}, f\left(r_{1}, \ldots, r_{n}\right)\right] f\left(r_{1}, \ldots, r_{n}\right) c^{\prime}=0 .
$$

Then by Corollary 2.12, $f\left(x_{1}, \ldots, x_{n}\right)^{2}$ is central valued in $R$ with $c, c^{\prime} \in C$ and $\left(c+c^{\prime}\right) a^{\prime} \in$ $C$. Since $a^{\prime} \notin C, c+c^{\prime}=0$ implying $F=0$, a contradiction.

Case II: Assume next that $d^{\prime}$ and $\delta$ are $C$-independent modulo inner derivations of $U$. Then applying Kharchenko's Theorem [14, we have from (2.5) that $U$ satisfies blended components

$$
\begin{aligned}
& c \sum_{i} f\left(r_{1}, \ldots, y_{i}, \ldots, r_{n}\right) f\left(r_{1}, \ldots, r_{n}\right)+\sum_{i} f\left(r_{1}, \ldots, y_{i}, \ldots, r_{n}\right) f\left(r_{1}, \ldots, r_{n}\right) c^{\prime} \\
= & f\left(r_{1}, \ldots, r_{n}\right) \sum_{i} f\left(r_{1}, \ldots, z_{i}, \ldots, r_{n}\right) .
\end{aligned}
$$

In particular, for $y_{1}=\cdots=y_{n}=0, U$ satisfies $f\left(r_{1}, \ldots, r_{n}\right) \sum_{i} f\left(r_{1}, \ldots, z_{i}, \ldots, r_{n}\right)=0$. In particular, $f\left(r_{1}, \ldots, r_{n}\right)^{2}=0$ for all $r_{1}, \ldots, r_{n} \in U$, implying $f\left(r_{1}, \ldots, r_{n}\right)=0$, a contradiction.

Lemma 2.15. Let $R$ be a noncommutative prime ring of characteristic different from 2 with Utumi quotient ring $U$ and extended centroid $C$, and $f\left(x_{1}, \ldots, x_{n}\right)$ a multilinear polynomial over $C$, which is not central valued on $R$. Suppose that $F$ and $H$ are two generalized derivations of $R$ and $G(x)=c x+x c^{\prime}$ for all $x \in R$, for some $c, c^{\prime} \in U$ is a nonzero inner generalized derivation of $R$, such that $F(G(f(r)) f(r))=f(r) H(f(r))$ for all $r=\left(r_{1}, \ldots, r_{n}\right) \in R^{n}$, then one of the following holds: 
(1) there exist $\lambda \in C$ and $a, b \in U$ such that $F(x)=\lambda x, G(x)=x a$ and $H(x)=b x$ for all $x \in R$ with $\lambda a=b$;

(2) there exist $\lambda, \alpha \in C$ and $p, q, u, v \in U$ such that $F(x)=\lambda x, G(x)=p x+x q$ and $H(x)=u x+x v$ for all $x \in R$ with $f\left(x_{1}, \ldots, x_{n}\right)^{2}$ being central valued in $R$ and $v-\lambda p=\lambda q-u=\alpha \in C$

(3) there exist $\lambda \in C$ and $a, p \in U$ such that $F(x)=a x, G(x)=p x$ and $H(x)=\lambda x$ for all $x \in R$ with ap $=\lambda$;

(4) there exist $\lambda \in C$ and $a, u \in U$ such that $F(x)=x a, G(x)=\lambda x$ and $H(x)=x u$ for all $x \in R$ with $a \lambda=u$;

(5) there exist $a, b, p, v \in U$ such that $F(x)=a x+x b, G(x)=p x$ and $H(x)=x v$ for all $x \in R$ with $f\left(x_{1}, \ldots, x_{n}\right)^{2}$ being central valued on $R$ and $a p+p b=v$.

Proof. In view of [16. Theorem 3], we may assume that there exist $a, b \in U$ and derivations $d^{\prime}, \delta$ of $U$ such that $F(x)=a x+d(x)$ and $H(x)=b x+\delta(x)$. Since $R$ and $U$ satisfy the same generalized polynomial identities (see [4]) as well as the same differential identities (see 15]), we may assume that

$$
a\left\{c f(r)^{2}+f(r) c^{\prime} f(r)\right\}+d\left\{c f(r)^{2}+f(r) c^{\prime} f(r)\right\}=f(r) b f(r)+f(r) \delta(f(r))
$$

for all $r=\left(r_{1}, \ldots, r_{n}\right) \in U^{n}$, where $d, \delta$ are two derivations on $U$.

If $F$ and $H$ both are inner generalized derivations of $R$, then by Lemma 2.11 we obtain our conclusions (1)-(5). Thus we assume that not both of $F$ and $H$ are inner. Then $d$ and $\delta$ cannot be both inner derivations of $U$. Now we consider the following two cases:

Case I: Assume that $d$ and $\delta$ are $C$-dependent modulo inner derivations of $U$, say $\alpha d+\beta \delta=a d_{q}$, where $\alpha, \beta \in C, q \in U$ and $a d_{q}(x)=[q, x]$ for all $x \in R$. If $\beta=0$, then $\alpha \neq 0$ and thus $d$ is inner. In this case conclusion follows by Lemma 2.14. Next we assume that $\beta \neq 0$. Then there exist some $\lambda \in C$ and $p \in U$ such that $\delta(x)=\lambda d(x)+[p, x]$ for all $x \in U$. The by 2.9 , $U$ satisfies

$$
\begin{aligned}
& a\left\{c f(r)^{2}+f(r) c^{\prime} f(r)\right\}+d(c) f(r)^{2}+c d(f(r)) f(r)+c f(r) d(f(r)) \\
& +d(f(r)) c^{\prime} f(r)+f(r) d\left(c^{\prime}\right) f(r)+f(r) c^{\prime} d(f(r)) \\
= & f(r) b f(r)+f(r) \lambda d(f(r))+f(r)[p, f(r)] .
\end{aligned}
$$

Since $f\left(r_{1}, \ldots, r_{n}\right)$ is a multilinear polynomial over $C$, we have $d\left(f\left(r_{1}, \ldots, r_{n}\right)\right)=$ $f^{d}\left(r_{1}, \ldots, r_{n}\right)+\sum_{i} f\left(r_{1}, \ldots, d\left(r_{i}\right), \ldots, r_{n}\right)$, where $f^{d}\left(r_{1}, \ldots, r_{n}\right)$ is the polynomials obtained from $f\left(r_{1}, \ldots, r_{n}\right)$ replacing each coefficients $\alpha_{\sigma}$ with $d\left(\alpha_{\sigma}\right)$. Thus by Kharchenko's 
Theorem [14, we can replace $d\left(f\left(r_{1}, \ldots, r_{n}\right)\right)$ by $f^{d}\left(r_{1}, \ldots, r_{n}\right)+\sum_{i} f\left(r_{1}, \ldots, y_{i}, \ldots, r_{n}\right)$ in 2.10 and then $U$ satisfies blended components

$$
\begin{aligned}
& c \sum_{i} f\left(r_{1}, \ldots, y_{i}, \ldots, r_{n}\right) f\left(r_{1}, \ldots, r_{n}\right)+c f\left(r_{1}, \ldots, r_{n}\right) \sum_{i} f\left(r_{1}, \ldots, y_{i}, \ldots, r_{n}\right) \\
& +\sum_{i} f\left(r_{1}, \ldots, y_{i}, \ldots, r_{n}\right) c^{\prime} f\left(r_{1}, \ldots, r_{n}\right)+f\left(r_{1}, \ldots, r_{n}\right) c^{\prime} \sum_{i} f\left(r_{1}, \ldots, y_{i}, \ldots, r_{n}\right) \\
= & f\left(r_{1}, \ldots, r_{n}\right) \lambda \sum_{i} f\left(r_{1}, \ldots, y_{i}, \ldots, r_{n}\right) .
\end{aligned}
$$

In particular, for $y_{1}=r_{1}$ and $y_{2}=\cdots=y_{n}=0, U$ satisfies

$$
(2 c-\lambda) f\left(r_{1}, \ldots, r_{n}\right)^{2}+f\left(r_{1}, \ldots, r_{n}\right)\left(2 c^{\prime}\right) f\left(r_{1}, \ldots, r_{n}\right)=0,
$$

which implies

$$
\left((2 c-\lambda) f\left(r_{1}, \ldots, r_{n}\right)+f\left(r_{1}, \ldots, r_{n}\right)\left(2 c^{\prime}\right)\right) f\left(r_{1}, \ldots, r_{n}\right)=0 .
$$

By Lemma 2.1, we conclude that $2 c^{\prime}=\lambda-2 c \in C$. Since $\operatorname{char}(R) \neq 2, c, c^{\prime} \in C$. Then by (2.11), $U$ satisfies

$$
\begin{aligned}
& \left(c+c^{\prime}\right) \sum_{i} f\left(r_{1}, \ldots, y_{i}, \ldots, r_{n}\right) f\left(r_{1}, \ldots, r_{n}\right) \\
+ & \left(c+c^{\prime}-\lambda\right) f\left(r_{1}, \ldots, r_{n}\right) \sum_{i} f\left(r_{1}, \ldots, y_{i}, \ldots, r_{n}\right)=0 .
\end{aligned}
$$

Replacing $y_{i}$ with $\left[q, x_{i}\right]$ for some $q^{\prime} \notin C$, we have

$$
\left(c+c^{\prime}\right)\left[q^{\prime}, f\left(r_{1}, \ldots, r_{n}\right)\right] f\left(r_{1}, \ldots, r_{n}\right)+\left(c+c^{\prime}-\lambda\right) f\left(r_{1}, \ldots, r_{n}\right)\left[q^{\prime}, f\left(r_{1}, \ldots, r_{n}\right)\right]=0
$$

that is,

$$
\left[\left(c+c^{\prime}\right) q^{\prime}, f\left(r_{1}, \ldots, r_{n}\right)\right] f\left(r_{1}, \ldots, r_{n}\right)+f\left(r_{1}, \ldots, r_{n}\right)\left[\left(c+c^{\prime}-\lambda\right) q^{\prime}, f\left(r_{1}, \ldots, r_{n}\right)\right]=0 .
$$

By Lemma 2.1, one of the following holds: (i) $\left(c+c^{\prime}\right) q^{\prime},\left(c+c^{\prime}-\lambda\right) q^{\prime} \in C$; in this case as $q^{\prime} \notin C, c+c^{\prime}=0$, implying $G=0$, a contradiction. (ii) $f\left(r_{1}, \ldots, r_{n}\right)^{2}$ is central valued and $\left(c+c^{\prime}-\lambda\right) q^{\prime}-\left(c+c^{\prime}\right) q^{\prime} \in C$, i.e., $\lambda q^{\prime} \in C$. In this case as $q^{\prime} \notin C, \lambda=0$. Thus $\lambda=2\left(c+c^{\prime}\right)=0$ implying $c+c^{\prime}=0$. Hence $G=0$, a contradiction.

Lemma 2.16. Let $R$ be a noncommutative prime ring of characteristic different from 2 with Utumi quotient ring $U$ and extended centroid $C$, and $f\left(x_{1}, \ldots, x_{n}\right)$ a multilinear polynomial over $C$, which is not central valued on $R$. Suppose that $F$ and $G$ are two generalized derivations of $R$ and $H(x)=b x+x b^{\prime}$ for all $x \in R$, for some $b, b^{\prime} \in U$ is a nonzero inner generalized derivation of $R$, such that $F(G(f(r)) f(r))=f(r) H(f(r))$ for all $r=\left(r_{1}, \ldots, r_{n}\right) \in R^{n}$, then one of the following holds: 
(1) there exist $\lambda \in C$ and $a, b \in U$ such that $F(x)=\lambda x, G(x)=x a$ and $H(x)=b x$ for all $x \in R$ with $\lambda a=b$;

(2) there exist $\lambda, \alpha \in C$ and $p, q, u, v \in U$ such that $F(x)=\lambda x, G(x)=p x+x q$ and $H(x)=u x+x v$ for all $x \in R$ with $f\left(x_{1}, \ldots, x_{n}\right)^{2}$ being central valued in $R$ and $v-\lambda p=\lambda q-u=\alpha \in C$

(3) there exist $\lambda \in C$ and $a, p \in U$ such that $F(x)=a x, G(x)=p x$ and $H(x)=\lambda x$ for all $x \in R$ with ap $=\lambda$;

(4) there exist $\lambda \in C$ and $a, u \in U$ such that $F(x)=x a, G(x)=\lambda x$ and $H(x)=x u$ for all $x \in R$ with $a \lambda=u$;

(5) there exist $a, b, p, v \in U$ such that $F(x)=a x+x b, G(x)=p x$ and $H(x)=x v$ for all $x \in R$ with $f\left(x_{1}, \ldots, x_{n}\right)^{2}$ being central valued on $R$ and $a p+p b=v$.

Proof. In view of [16. Theorem 3], we may assume that there exist $a, b \in U$ and derivations $d^{\prime}, \delta$ of $U$ such that $F(x)=c x+d(x)$ and $G(x)=a x+d^{\prime}(x)$. Since $R$ and $U$ satisfy the same generalized polynomial identities (see [4]) as well as the same differential identities (see 15]), we may assume that

$$
c\left\{a f(r)^{2}+d^{\prime}(f(r)) f(r)\right\}+d\left\{a f(r)^{2}+d^{\prime}(f(r)) f(r)\right\}=f(r) b f(r)+f(r)^{2} b^{\prime}
$$

for all $r=\left(r_{1}, \ldots, r_{n}\right) \in U^{n}$, where $d, d^{\prime}$ are two derivations on $U$.

If $d$ or $d^{\prime}$ is inner, then $F$ or $G$ is inner and then by Lemmas 2.14 and 2.15 , we obtain our conclusions (1)-(5). Thus we assume that both of $d$ and $d^{\prime}$ are outer. Now we consider the following two cases:

Case I: Assume that $d$ and $d^{\prime}$ are $C$-dependent modulo inner derivations of $U$, then $d=\alpha d^{\prime}+a d_{p^{\prime}}$. Then 2.12 becomes

$$
\begin{aligned}
& c\left\{a f(r)^{2}+d^{\prime}(f(r)) f(r)\right\}+\alpha d^{\prime}\left\{a f(r)^{2}+d^{\prime}(f(r)) f(r)\right\}+\left[p^{\prime}, a f(r)^{2}+d^{\prime}(f(r)) f(r)\right] \\
= & f(r) b f(r)+f(r)^{2} b^{\prime} .
\end{aligned}
$$

We know that $d^{\prime}\left(f\left(r_{1}, \ldots, r_{n}\right)\right)=f^{d^{\prime}}\left(r_{1}, \ldots, r_{n}\right)+\sum_{i} f\left(r_{1}, \ldots, d^{\prime}\left(r_{i}\right), \ldots, r_{n}\right)$, and

$$
\begin{aligned}
d^{\prime 2}\left(f\left(r_{1}, \ldots, r_{n}\right)\right)= & f^{d^{\prime 2}}\left(r_{1}, \ldots, r_{n}\right)+2 \sum_{i} f^{d^{\prime}}\left(r_{1}, \ldots, d^{\prime}\left(r_{i}\right), \ldots, r_{n}\right) \\
& +\sum_{i} f\left(r_{1}, \ldots, d^{\prime 2}\left(r_{i}\right), \ldots, r_{n}\right) \\
& +\sum_{i \neq j} f\left(r_{1}, \ldots, d^{\prime}\left(r_{i}\right), \ldots, d^{\prime}\left(r_{j}\right), \ldots, r_{n}\right) .
\end{aligned}
$$


By applying Kharchenko's Theorem [14], we can replace $d\left(f\left(r_{1}, \ldots, r_{n}\right)\right)$ with $f^{d}\left(r_{1}, \ldots, r_{n}\right)$ $+\sum_{i} f\left(r_{1}, \ldots, y_{i}, \ldots, r_{n}\right)$ and $d^{\prime 2}\left(f\left(r_{1}, \ldots, r_{n}\right)\right)$ with

$$
\begin{aligned}
& f^{d^{\prime 2}}\left(r_{1}, \ldots, r_{n}\right)+2 \sum_{i} f^{d^{\prime}}\left(r_{1}, \ldots, y_{i}, \ldots, r_{n}\right) \\
+ & \sum_{i} f\left(r_{1}, \ldots, t_{i}, \ldots, r_{n}\right)+\sum_{i \neq j} f\left(r_{1}, \ldots, y_{i}, \ldots, y_{j}, \ldots, r_{n}\right)
\end{aligned}
$$

in 2.13 and then $U$ satisfies blended component

$$
\alpha \sum_{i} f\left(r_{1}, \ldots, t_{i}, \ldots, r_{n}\right) f\left(r_{1}, \ldots, r_{n}\right)=0
$$

This implies $\alpha f\left(x_{1}, \ldots, x_{n}\right)^{2}=0$, implying $\alpha=0$. Then $d$ is inner, a contradiction.

Case II: Assume that $d$ and $d^{\prime}$ are $C$-independent modulo inner derivations of $U$.

Then applying Kharchenko's Theorem [14] to 2.12), we can replace

$$
\begin{gathered}
d^{\prime}\left(f\left(r_{1}, \ldots, r_{n}\right)\right)=f^{d^{\prime}}\left(r_{1}, \ldots, r_{n}\right)+\sum_{i} f\left(r_{1}, \ldots, y_{i}, \ldots, r_{n}\right), \\
d\left(f\left(r_{1}, \ldots, r_{n}\right)\right)=f^{d}\left(r_{1}, \ldots, r_{n}\right)+\sum_{i} f\left(r_{1}, \ldots, t_{i}, \ldots, r_{n}\right),
\end{gathered}
$$

and

$$
\begin{aligned}
d d^{\prime}\left(f\left(r_{1}, \ldots, r_{n}\right)\right)= & f^{d d^{\prime}}\left(r_{1}, \ldots, r_{n}\right)+\sum_{i} f^{\delta}\left(r_{1}, \ldots, y_{i}, \ldots, r_{n}\right) \\
& +\sum_{i} f^{d^{\prime}}\left(r_{1}, \ldots, t_{i}, \ldots, r_{n}\right)+\sum_{i \neq j} f\left(r_{1}, \ldots, y_{i}, \ldots, t_{j}, \ldots, r_{n}\right) \\
& +\sum_{i} f\left(r_{1}, \ldots, w_{i}^{\prime}, \ldots, r_{n}\right) .
\end{aligned}
$$

Then $U$ satisfies blended component $\sum_{i} f\left(r_{1}, \ldots, w_{i}^{\prime}, \ldots, r_{n}\right) f\left(r_{1}, \ldots, r_{n}\right)=0$. In particular, $f\left(r_{1}, \ldots, r_{n}\right)^{2}=0$ implying $f\left(r_{1}, \ldots, r_{n}\right)=0$, a contradiction.

Proof of Theorem 1.1. If any one of $F$ or $G$ or $H$ is inner, then conclusion follows by Lemmas 2.14, 2.15 and 2.16 .

Thus we assume that $F, G$ and $H$ are all outer generalized derivations of $R$. Then by [16], we have $F(x)=c x+d(x), G(x)=a x+d^{\prime}(x)$ and $H(x)=b x+\delta(x)$ for some $a, b, c \in U$ and $d, d^{\prime}, \delta$ are three derivations of $U$. By hypothesis, we have

$$
c\left\{a f(r)^{2}+d^{\prime}(f(r)) f(r)\right\}+d\left\{a f(r)^{2}+d^{\prime}(f(r)\} f(r)\right)=f(r) b f(r)+f(r) \delta(f(r))
$$

for all $r=\left(r_{1}, \ldots, r_{n}\right) \in U^{n}$. Now we consider the following two cases:

Case 1: Let $d^{\prime}$ and $\delta$ be $C$-dependent modulo inner derivations of $U$, i.e., $\alpha d^{\prime}+\beta \delta=$ $a d_{p^{\prime}}$. 
Now $\alpha=0$ implies that $\delta$ is inner, a contradiction as $H$ cannot be inner. Thus $\alpha \neq 0$. Then $d^{\prime}=\lambda \delta+a d_{p}$, where $\lambda=-\beta \alpha^{-1} \in C$ and $p=p^{\prime} \alpha^{-1} \in U$. Therefore, 2.14) gives

$$
\begin{aligned}
& c\left\{a f(r)^{2}+\lambda \delta(f(r)) f(r)+[p, f(r)] f(r)\right\}+d\left(a f(r)^{2}+\lambda \delta(f(r)) f(r)+[p, f(r)] f(r)\right) \\
= & f(r) b f(r)+f(r) \delta(f(r))
\end{aligned}
$$

for all $r=\left(r_{1}, \ldots, r_{n}\right) \in U^{n}$, that is,

$$
\begin{aligned}
& c\left(a f(r)^{2}+\lambda \delta(f(r)) f(r)+[p, f(r)] f(r)\right)+d\left(a f(r)^{2}+[p, f(r)] f(r)\right) \\
& +d(\lambda) \delta(f(r)) f(r)+\lambda(d \delta)(f(r)) f(r)+\lambda \delta(f(r)) d(f(r)) \\
= & f(r) b f(r)+f(r) \delta(f(r))
\end{aligned}
$$

for all $r=\left(r_{1}, \ldots, r_{n}\right) \in U^{n}$. We know that

$$
d\left(f\left(r_{1}, \ldots, r_{n}\right)\right)=f^{d}\left(r_{1}, \ldots, r_{n}\right)+\sum_{i} f\left(r_{1}, \ldots, d\left(r_{i}\right), \ldots, r_{n}\right)
$$

and

$$
\begin{aligned}
\delta d\left(f\left(r_{1}, \ldots, r_{n}\right)\right)= & f^{\delta d}\left(r_{1}, \ldots, r_{n}\right)+\sum_{i} f^{d}\left(r_{1}, \ldots, \delta\left(r_{i}\right), \ldots, r_{n}\right) \\
& +\sum_{i} f^{\delta}\left(r_{1}, \ldots, d\left(r_{i}\right), \ldots, r_{n}\right)+\sum_{i} f\left(r_{1}, \ldots, \delta d\left(r_{i}\right), \ldots, r_{n}\right) \\
& +\sum_{i} f\left(r_{1}, \ldots, \delta\left(r_{i}\right), \ldots, d\left(r_{j}\right), \ldots, r_{n}\right) .
\end{aligned}
$$

Let $\delta$ and $d$ be $C$-independent modulo inner derivations of $U$. By applying Kharchenko's Theorem 14 to 2.15, we can replace $d\left(f\left(r_{1}, \ldots, r_{n}\right)\right)$ with $f^{d}\left(r_{1}, \ldots, r_{n}\right)$

$+\sum_{i} f\left(r_{1}, \ldots, y_{i}, \ldots, r_{n}\right)$ and $\delta d\left(f\left(r_{1}, \ldots, r_{n}\right)\right)$ with

$$
\begin{aligned}
& f^{\delta d}\left(r_{1}, \ldots, r_{n}\right)+\sum_{i} f^{d}\left(r_{1}, \ldots, s_{i}, \ldots, r_{n}\right)+\sum_{i} f^{\delta}\left(r_{1}, \ldots, y_{i}, \ldots, r_{n}\right) \\
+ & \sum_{i} f\left(r_{1}, \ldots, t_{i}, \ldots, r_{n}\right)+\sum_{i} f\left(r_{1}, \ldots, s_{i}, \ldots, y_{j}, \ldots, r_{n}\right)
\end{aligned}
$$

in 2.15) and then $U$ satisfies blended component

$$
\lambda \sum_{i} f\left(r_{1}, \ldots, t_{i}, \ldots, r_{n}\right) f\left(r_{1}, \ldots, r_{n}\right)=0
$$

In particular, for $t_{1}=r_{1}$ and $t_{2}=\cdots=t_{n}=0$ in 2.16 , we have $\lambda f\left(r_{1}, \ldots, r_{n}\right)^{2}=0$. If $\lambda \neq 0$, then $f\left(r_{1}, \ldots, r_{n}\right)^{2}=0$ which implies $f\left(r_{1}, \ldots, r_{n}\right)=0$ for all $r_{1}, \ldots, r_{n} \in U$ (see [5]), a contradiction. Thus $\lambda=0$. In this case $G$ becomes inner, a contradiction. 
Now let $\delta$ and $d$ be $C$-dependent, i.e., $\alpha_{1} \delta+\beta_{1} d=a d_{q^{\prime}}$. Now, $\alpha_{1}=0$, implies $d$ is inner, a contradiction. Thus $\alpha_{1} \neq 0$ and so $\delta=\mu d+[q, x]$ for some $\mu \in C$ and $q \in U$. Then by 2.15), $U$ satisfies

$$
\begin{aligned}
& c\left(a f(r)^{2}+\lambda \mu d(f(r)) f(r)+\lambda[q, f(r)] f(r)+[p, f(r)] f(r)\right) \\
& +d\left(a f(r)^{2}+[p, f(r)] f(r)\right)+d(\lambda) \mu d(f(r)) f(r)+d(\lambda)[q, f(r)] f(r) \\
& +\lambda d(\mu d(f(r))+[q, f(r)]) f(r)+\lambda(\mu d(f(r))+[q, f(r)]) d(f(r)) \\
= & f(r) b f(r)+f(r)(\mu d(f(r))+[q, f(r)])
\end{aligned}
$$

for all $r=\left(r_{1}, \ldots, r_{n}\right) \in U^{n}$.

Since $d\left(f\left(r_{1}, \ldots, r_{n}\right)\right)=f^{d}\left(r_{1}, \ldots, r_{n}\right)+\sum_{i} f\left(r_{1}, \ldots, d\left(r_{i}\right), \ldots, r_{n}\right)$ and

$$
\begin{aligned}
d^{2}\left(f\left(r_{1}, \ldots, r_{n}\right)\right)= & f^{d^{2}}\left(r_{1}, \ldots, r_{n}\right)+2 \sum_{i} f^{d}\left(r_{1}, \ldots, d\left(r_{i}\right), \ldots, r_{n}\right) \\
& +\sum_{i} f\left(r_{1}, \ldots, d^{2}\left(r_{i}\right), \ldots, r_{n}\right) \\
& +\sum_{i \neq j} f\left(r_{1}, \ldots, d\left(r_{i}\right), \ldots, d\left(r_{j}\right), \ldots, r_{n}\right),
\end{aligned}
$$

by applying Kharchenko's Theorem [14], we can replace $d\left(f\left(r_{1}, \ldots, r_{n}\right)\right)$ with $f^{d}\left(r_{1}, \ldots, r_{n}\right)$ $+\sum_{i} f\left(r_{1}, \ldots, y_{i}, \ldots, r_{n}\right)$ and $d^{2}\left(f\left(r_{1}, \ldots, r_{n}\right)\right)$ with

$$
\begin{aligned}
d^{2}\left(f\left(r_{1}, \ldots, r_{n}\right)\right)= & f^{d^{2}}\left(r_{1}, \ldots, r_{n}\right)+2 \sum_{i} f^{d}\left(r_{1}, \ldots, y_{i}, \ldots, r_{n}\right) \\
& +\sum_{i} f\left(r_{1}, \ldots, t_{i}, \ldots, r_{n}\right)+\sum_{i \neq j} f\left(r_{1}, \ldots, y_{i}, \ldots, y_{j}, \ldots, r_{n}\right),
\end{aligned}
$$

and then $U$ satisfies blended component

$$
\lambda \mu \sum_{i} f\left(r_{1}, \ldots, t_{i}, \ldots, r_{n}\right) f\left(r_{1}, \ldots, r_{n}\right)=0 .
$$

In particular, $\lambda \mu f\left(r_{1}, \ldots, r_{n}\right)^{2}=0$. This implies $\lambda \mu=0$ and so either $\lambda=0$ or $\mu=0$. Now $\lambda=0$ gives $G$ is inner, a contradiction. Again $\mu=0$, gives $H$ is inner, a contradiction.

Case 2: Let $d^{\prime}$ and $\delta$ be $C$-independent modulo inner derivations of $U$. We divide the proof into two subcases.

Subcase $i$. Let $d, d^{\prime}$ and $\delta$ be $C$-independent modulo inner derivations of $U$. In this case we rewrite (2.14) as

$$
\begin{aligned}
& c\left(a f(r)^{2}+d^{\prime}(f(r)) f(r)\right)+d(a) f(r)^{2}+a d(f(r)) f(r) \\
& +a f(r) d(f(r))+d d^{\prime}(f(r)) f(r)+d^{\prime}(f(r)) d(f(r)) \\
= & f(r) b f(r)+f(r) \delta(f(r))
\end{aligned}
$$


for all $r=\left(r_{1}, \ldots, r_{n}\right) \in U^{n}$.

By applying Kharchenko's Theorem [14, we can replace $d d^{\prime}\left(f\left(x_{1}, \ldots, x_{n}\right)\right)$ by

$$
\begin{aligned}
& f^{d d^{\prime}}\left(r_{1}, \ldots, r_{n}\right)+\sum_{i} f^{d^{\prime}}\left(r_{1}, \ldots, x_{i}, \ldots, r_{n}\right)+\sum_{i} f^{d}\left(r_{1}, \ldots, t_{i}, \ldots, r_{n}\right) \\
+ & \sum_{i \neq j} f\left(r_{1}, \ldots, t_{i}, \ldots, x_{j}, \ldots, r_{n}\right)+\sum_{i} f\left(r_{1}, \ldots, w_{i}, \ldots, r_{n}\right)
\end{aligned}
$$

in above equality and then $U$ satisfies the blended component

$$
\sum_{i} f\left(r_{1}, \ldots, w_{i}, \ldots, r_{n}\right) f\left(r_{1}, \ldots, r_{n}\right)=0 .
$$

In particular for $w_{1}=r_{1}$ and $w_{2}=\cdots=w_{n}=0, U$ satisfies $f\left(r_{1}, \ldots, r_{n}\right)^{2}=0$ implying $f\left(r_{1}, \ldots, r_{n}\right)=0$, a contradiction.

Subcase ii. Let $d, d^{\prime}$ and $\delta$ be $C$-dependent modulo inner derivations of $U$, i.e., $\alpha_{1} d+$ $\alpha_{2} d^{\prime}+\alpha_{3} \delta=a d_{a^{\prime}}$ for some $\alpha_{1}, \alpha_{2}, \alpha_{3} \in C$. Then $\alpha_{1} \neq 0$, otherwise $d^{\prime}$ and $\delta$ are $C$ dependent modulo inner derivation of $U$, a contradiction. Then we can write $d=\beta_{1} d^{\prime}+$ $\beta_{2} \delta+a d_{a^{\prime \prime}}$ for some $\beta_{1}, \beta_{2} \in C$ and $a^{\prime \prime} \in U$. Then by (2.14), we have

$$
\begin{aligned}
& c\left\{a f(r)^{2}+d^{\prime}(f(r)) f(r)\right\}+\beta_{1} d^{\prime}\left\{a f(r)^{2}+d^{\prime}(f(r)) f(r)\right\} \\
& +\beta_{2} \delta\left\{a f(r)^{2}+d^{\prime}(f(r)) f(r)\right\}+\left[a^{\prime \prime}, a f(r)^{2}+d^{\prime}(f(r)) f(r)\right] \\
= & f(r) b f(r)+f(r) \delta(f(r))
\end{aligned}
$$

for all $r=\left(r_{1}, \ldots, r_{n}\right) \in U^{n}$.

Using Kharchenko's Theorem [14, we substitute the following values in (2.18)

$$
\begin{aligned}
d^{\prime}\left(f\left(r_{1}, \ldots, r_{n}\right)\right)= & f^{d^{\prime}}\left(r_{1}, \ldots, r_{n}\right)+\sum_{i} f\left(r_{1}, \ldots, y_{i}, \ldots, r_{n}\right), \\
\delta\left(f\left(r_{1}, \ldots, r_{n}\right)\right)= & f^{\delta}\left(r_{1}, \ldots, r_{n}\right)+\sum_{i} f\left(r_{1}, \ldots, t_{i}, \ldots, r_{n}\right), \\
\delta d^{\prime}\left(f\left(r_{1}, \ldots, r_{n}\right)\right)= & f^{\delta d^{\prime}}\left(r_{1}, \ldots, r_{n}\right)+\sum_{i} f^{\delta}\left(r_{1}, \ldots, y_{i}, \ldots, r_{n}\right) \\
& +\sum_{i} f^{d^{\prime}}\left(r_{1}, \ldots, t_{i}, \ldots, r_{n}\right)+\sum_{i \neq j} f\left(r_{1}, \ldots, y_{i}, \ldots, t_{j}, \ldots, r_{n}\right) \\
& +\sum_{i} f\left(r_{1}, \ldots, w_{i}^{\prime}, \ldots, r_{n}\right), \\
d^{\prime 2}\left(f\left(r_{1}, \ldots, r_{n}\right)\right)= & f^{d^{\prime 2}}\left(r_{1}, \ldots, r_{n}\right)+2 \sum_{i} f^{d^{\prime}}\left(r_{1}, \ldots, y_{i}, \ldots, r_{n}\right) \\
& +\sum_{i} f\left(r_{1}, \ldots, z_{i}^{\prime}, \ldots, r_{n}\right)+\sum_{i \neq j} f\left(r_{1}, \ldots, y_{i}, \ldots, y_{j}, \ldots, r_{n}\right) .
\end{aligned}
$$

Therefore, $U$ satisfies the blended components

$$
\beta_{1} \sum_{i} f\left(r_{1}, \ldots, z_{i}^{\prime}, \ldots, r_{n}\right) f\left(r_{1}, \ldots, r_{n}\right)=0
$$


and

$$
\beta_{2} \sum_{i} f\left(r_{1}, \ldots, w_{i}^{\prime}, \ldots, r_{n}\right) f\left(r_{1}, \ldots, r_{n}\right)=0 .
$$

If $\beta_{1} \neq 0$, then from above, $U$ satisfies

$$
\sum_{i} f\left(r_{1}, \ldots, z_{i}^{\prime}, \ldots, r_{n}\right) f\left(r_{1}, \ldots, r_{n}\right)=0 .
$$

This is same as 2.17) and hence by same argument as above, it leads to a contradiction. Thus we conclude that $\beta_{1}=0$. Similarly, from above relation, we conclude that $\beta_{2}=0$. Then $d$ is inner, contradicting with the fact that $F$ is outer. This complete the proof of the theorem.

\section{References}

[1] N. Argaç and V. De Filippis, Actions of generalized derivations on multilinear polynomials in prime rings, Algebra Colloq. 18 (2011), Special Issue no. 1, 955-964.

[2] K. I. Beidar, W. S. Martindale III and A. V. Mikhalev, Rings with Generalized Identities, Monographs and Textbooks in Pure and Applied Mathematics 196, Marcel Dekker, New York, 1996.

[3] L. Carini and V. de Filippis, Centralizers of generalized derivations on multilinear polynomials in prime rings, Sib. Math. J. 53 (2012), no. 6, 1051-1060.

[4] C.-L. Chuang, GPIs having coefficients in Utumi quotient rings, Proc. Amer. Math. Soc. 103 (1988), no. 3, 723-728.

[5] C.-L. Chuang and T.-K. Lee, Rings with annihilator conditions on multilinear polynomials, Chinese J. Math. 24 (1996), no. 2, 177-185.

[6] V. De Filippis and O. M. Di Vincenzo, Vanishing derivations and centralizers of generalized derivations on multilinear polynomials, Comm. Algebra 40 (2012), no. 6, 1918-1932.

[7] B. Dhara, Generalized derivations acting on multilinear polynomials in prime rings, Czechoslovak Math. J. 68 (2018), no. 1, 95-119.

[8] B. Dhara and N. Argaç, Generalized derivations acting on multilinear polynomials in prime rings and Banach algebras, Commun. Math. Stat. 4 (2016), no. 1, 39-54.

[9] B. Dhara, N. Argac and E. Albas, Vanishing derivations and co-centralizing generalized derivations on multilinear polynomials in prime rings, Comm. Algebra 44 (2016), no. 5, 1905-1923. 
[10] B. Dhara and V. De Filippis, b-generalized derivations acting on multilinear polynomials in prime rings, Algebra Colloq. 25 (2018), no. 4, 681-700.

[11] T. S. Erickson, W. S. Martindale III and J. M. Osborn, Prime nonassociative algebras, Pacific J. Math. 60 (1975), no. 1, 49-63.

[12] C. Faith and Y. Utumi, On a new proof of Litoff's theorem, Acta Math. Acad. Sci. Hungar. 14 (1963), 369-371.

[13] N. Jacobson, Structure of Rings, American Mathematical Society Colloquium Publications 37, American Mathematical Society, Providence, R.I., 1964.

[14] V. K. Kharchenko, Differential identities of prime rings, Algebra Logic 17 (1978), no. $2,155-168$.

[15] T.-K. Lee, Semiprime rings with differential identities, Bull. Inst. Math. Acad. Sinica 20 (1992), no. 1, 27-38.

[16] Generalized derivations of left faithful rings, Comm. Algebra 27 (1999), no. 8, 4057-4073.

[17] U. Leron, Nil and power-central polynomials in rings, Trans. Amer. Math. Soc. 202 (1975), 97-103.

[18] W. S. Martindale III, Prime rings satisfying a generalized polynomial identity, J. Algebra 12 (1969), 576-584.

Basudeb Dhara

Department of Mathematics, Belda College, Belda, Paschim Medinipur, 721424, W.B., India

E-mail address: basu_dhara@yahoo.com

Nripendu Bera and Sukhendu Kar

Department of Mathematics, Jadavpur University, Kolkata, 700032, W.B., India

E-mail address: nripendub@gmail.com, karsukhendu@yahoo.co.in

Brahim Fahid

Superior School of Technology, Ibn Tofail University, Kenitra, Morocco

E-mail address: brahim.fahid@uit.ac.ma 